\title{
SOME REMARKS ON \\ TUBULAR NEIGHBORHOODS AND GLUING IN MORSE-FLOER HOMOLOGY
}

\author{
MAURIZIO RINALDI \\ Dipartimento di Scienze Matematiche, Università degli Studi di Trieste \\ Piazzale Europa, 1, 34100 Trieste, Italy \\ E-mail: rinaldi@univ.trieste.it \\ KRZYSZTOF P. RYBAKOWSKI \\ Fachbereich Mathematik, Universität Rostock \\ Universitätsplatz 1, 18055 Rostock, Germany \\ E-mail: krzysztof.rybakowski@mathematik.uni-rostock.de
}

\begin{abstract}
We discuss the gluing principle in Morse-Floer homology and show that there is a gap in the traditional proof of the converse gluing theorem. We show how this gap can be closed by the use of a uniform tubular neighborhood theorem. The latter result is only stated here. Details are given in the authors' paper, Tubular neighborhoods and the Gluing Principle in Floer homology theory, to appear.
\end{abstract}

1. Introduction. Let $X$ be a locally compact metric space and $\pi$ be a local flow on $X$. If $S$ is a compact isolated invariant set for $\pi$ then the Conley index $h(S)$ of $S$ is defined as the homotopy type of the pointed space $N_{1} / N_{0}$, where $\left(N_{1}, N_{0}\right)$ is a so called index pair for $S$. One could e.g. choose $N_{1}=B$, where $B$ is an isolating block for $S$, i.e. a compact neighborhood of $S$ whose boundary is 'transversal' to the flow. In this case one would define $N_{0}$ to be the set $B^{-}$of exit and bounce off points of $B$.

Applying an arbitrary homology or cohomology functor $H$ to $h(S)$ one obtains a (co)homological Conley index $H(h(S))$ of $S$.

Conley index is an extremely useful invariant which has had numerous applications to the qualitative study of ordinary differential equations in finite dimensional spaces. On the other hand, infinite dimensional problems can only be treated in those special cases in which a finite dimensional reduction is possible. This is due to the fact that in the

1991 Mathematics Subject Classification: Primary 53C20; Secondary 58F09, 58F05.

This research was supported by MURST $40 \%$ and $60 \%$.

The paper is in final form and no version of it will be published elsewhere. 
original setting of the theory the local compactness of the phase space $X$ (and also the two-sidedness of the flow) is crucial (cf Conley's monograph [3]). Thus Conley's theory as such is not applicable to various infinite dimensional flows generated by certain partial or functional differential equations since in such cases the phase space is not locally compact and the corresponding flow is only one-sided, i.e. a semiflow.

Therefore efforts have been made to extend the Conley index to semiflows $\pi$ on (metric) spaces $X$ which are not necessarily locally compact. The first such extension was made by Rybakowski in [10] and [11] (cf. also the paper [13] by Rybakowski and Zehnder and the book [12] by Rybakowski). In that theory it is assumed that the given isolated invariant set has an (end-point) admissible isolated neighborhood, i.e. an isolating neighborhood $N$ satisfying the following property:

Whenever $\left(x_{n}\right)$ is a sequence in $X$ and $\left(t_{n}\right)$ is a sequence of positive real numbers with $t_{n} \rightarrow \infty$ and if $x_{n} \pi\left[0, t_{n}\right] \subset N$ for all $n \in \mathbb{N}$, then the end-point sequence $\left(x_{n} \pi t_{n}\right)$ has a convergent subsequence.

For such isolated invariant sets a Conley index can be defined which enjoys all the properties of the original theory. End-point admissibility is satisfied for semilinear parabolic differential equations on bounded domains and for functional differential equations with finite delays. More generally, bounded sets are end-point admissible whenever the time-one map of the given semiflow is a conditional $\alpha$-contraction.

Later on the Conley index theory was even further generalized by Benci [2]. In his work the isolated neighborhood is only required to be mid-point admissible i.e. to have the property that

Whenever $\left(x_{n}\right)$ is a sequence in $X$ and $\left(t_{n}\right)$ is a sequence of positive real numbers with $t_{n} \rightarrow \infty$ and if $x_{n} \pi\left[0, t_{n}\right] \subset N$ for all $n \in \mathbb{N}$, then the mid-point sequence $\left(x_{n} \pi\left(t_{n} / 2\right)\right)$ has a convergent subsequence.

Clearly, mid-point admissibility is weaker than end-point admissibility. The resulting Conley index theory has, in particular, been applied to some ordinary differential equations of the form

$$
\dot{u}=-\nabla f(u)
$$

where $X=M$ is an infinite dimensional Riemannian manifold and $f: M \rightarrow \mathbb{R}$ is a $C^{2}$ - function satisfying the Palais-Smale condition and some additional assumptions (see $[2])$.

To illustrate the difference between end-point and mid-point admissibility let us consider a very simple example. Let $q \in M$ be a hyperbolic equilibrium point of equation (1.1), i.e. a hyperbolic critical point of $f$. Then $\{q\}$ is an isolated invariant set for (1.1). This set admits an end-point admissible isolating neighborhood if and only if the unstable manifold $W^{u}(q)$ of $q$ is finite dimensional. On the other hand every sufficiently small closed neighborhood of $\{q\}$ is mid-point admissible. Consequently, if $W^{u}(q)$ of $q$ is finite dimensional then both of the above-mentioned extensions of Conley theory can be applied and yield the same index of $\{q\}$.

Now suppose that $W^{u}(q)$ is infinite dimensional. In this case only Benci's theory is applicable. The resulting Conley index $h(\{q\})$ is the homotopy type of $D / \partial D$ where $D$ is the closed unit ball in the (infinite dimensional) tangent space of $W^{u}(q)$ at $q$. However, in 
every infinite dimensional normed space the unit sphere is a strong deformation retract of the closed unit ball (see e.g. [4, p. 66]), so the homotopy type of $D / \partial D$ is $\overline{0}$, where we denote by $\overline{0}$ the homotopy type of a one-point space. Thus

$$
h(\{q\})=\overline{0} .
$$

Now suppose, more generally, that $S$ is an isolated invariant set of the equation (1.1) which has a mid-point admissible isolating neighborhood and which contains only finitely many equilibrium points, say $q_{1}, \ldots, q_{n}$. We may assume that $f\left(q_{k}\right) \leq f\left(q_{k+1}\right)$ for $k=1, \ldots$, $n-1$. Then the following Morse equation holds:

$$
\sum_{k=1}^{n} p\left(t, h\left(\left\{q_{k}\right\}\right)\right)=p(t, h(S))+(1+t) Q(t) .
$$

Here, for a given isolated invariant set $J$ having Conley index $h(J), p(\cdot, h(J))$ is the formal power series

$$
p(t, h(J))=\sum_{l=0}^{\infty} \beta_{l} t^{l} \quad \text { with } \beta_{l}=\operatorname{rank} H^{l}(h(J)) \text { for all nonnegative integers } l .
$$

Moreover, $Q$ is a formal power series with only nonnegative coefficients.

Suppose that all equilibria in $S$ are hyperbolic. Then by what we said above only equilibria with finite dimensional unstable manifolds can have nonzero contribution to (1.2). Therefore if all equilibria in $S$ have infinite dimensional unstable manifolds (a situation which occurs in applications to wave equations or to periodic solutions of Hamiltonian systems) then the left hand side of (1.2) is zero. This implies that the cohomological Conley index of $S$ is zero. Thus in such cases the theory, even if applicable, gives only trivial index. Of course an analogous result holds for homology.

The point here is that Conley index measures the whole (infinite dimensional) unstable manifold $W^{u}(q)$ of an equilibrium point $q \in S$ while the invariant set $S$ is only the union of the set $R$ of its equilibria and all the sets $M_{x, y} \cap S, x, y \in R$, where $M_{x, y}$ is the set of all orbits of (1.1) connecting $x$ to $y$. Consequently $S$ may well be of finite dimension.

Therefore one may ask how much of the flow 'around' $S$ is really needed to determine the (co)homological Conley index of $S$.

An answer to this question was given by Floer [6] for the flow defined by equation (1.1), where $X=M$ is a finite dimensional Riemannian manifold and $f: M \rightarrow \mathbb{R}$ is a Morse-Smale function. For this case Floer gives the following alternative description of the cohomological Conley index:

Let $S$ be a compact invariant set of (1.1). Given a ring $\mathbb{F}$ and a nonnegative integer $\mu$ let $C^{\mu}$ be the free $\mathbb{F}$-module generated by all equilibria in $S$ having Morse index $\mu$. Moreover, set $C^{\mu}$ to be the trivial module for negative integers $\mu$.

Note that up to time-translations the orbits in $S$ connecting $x \in C^{\mu+1}$ to $y \in C^{\mu}$ lie on a compact zero dimensional manifold and so they are finite in number. Let $n(x, y) \in \mathbb{Z}$ be their number in some oriented sense. The precise definition of the orientation is immaterial here. Suffice it to say that in order to define this orientation we need the linearized flow along each connection from $x$ to $y$ lying in $S$. In the special case $\mathbb{F}=\mathbb{Z}_{2}$ the number $n(x, y)$ is just the $\bmod \mathbb{Z}_{2}$-number of connections from $x$ to $y$ lying in $S$. 
For $\mu \geq 0$ define the $\mathbb{F}$-linear operator

$$
\delta_{\mu}: C^{\mu} \rightarrow C^{\mu+1}
$$

by

$$
\delta_{\mu} y=\sum_{x \in C^{\mu+1}} n(x, y) x, \quad y \in C^{\mu} .
$$

Furthermore, let $\delta_{\mu}=0$ for $\mu<0$. Floer proves that this defines a coboundary operator (i.e. that $\delta_{\mu+1} \circ \delta_{\mu}=0$ for all $\left.\mu \in \mathbb{Z}\right)$ so $\left(\bigoplus_{\mu \in \mathbb{Z}} C^{\mu}, \bigoplus_{\mu \in \mathbb{Z}} \delta_{\mu}\right)$ is a cochain complex, called the Witten complex of $S$. Floer further shows that the Witten complex of $S$ is naturally isomorphic to the cohomological index $H(h(S))$ where $H$ is any cohomology theory with coefficients in $\mathbb{F}$. Thus in order to define the cohomological Conley index of $S$ in the Morse-Smale case we only need $S$ itself and the linearized flow along each orbit in $S$. Of course an analogous result can be stated and proved for homology.

Floer's result is related to cellular (co)homology and it was implicitly proved before by Milnor, Smale and Thom. However, it was Floer who noticed that the Witten complex of an invariant set $S$ can sometimes be defined and give nonzero (co)homology even in in situations where all critical points are strongly indefinite, i.e. have infinite-dimensional stable and unstable manifolds.

Floer constructed such a (co)homology index both for the symplectic action defined on the space of loops in a finite-dimensional symplectic manifold ([6] and [8]), which led to a proof of a generalized Arnold conjecture, and for the Chern-Simons action defined on the space of connections on a $S U(2)$-bundle over an homology sphere ([7]). In the latter case the Euler-Poincaré characteristic of the corresponding homology is the Casson invariant.

To develop the theory it is first necessary to define a 'good' notion of (relative) Morse indices for the critical points of the given strongly indefinite function $f$. After that one can define the operator $\delta_{\mu}$ as above. Then it must be shown that this really is a coboundary operator. It is at this point that the notion of trajectory gluing comes into play. More specifically, given three equilibria $x, y$ and $z \in M$ of (1.1), gluing establishes a relation between $M_{x, y} \times M_{y, z}$, and $M_{x, z}$. Intuitively, one would like to 'glue' a connection $u \in M_{x, y}$ with a connection $v \in M_{y, z}$ so as to obtain a connection in $M_{x, z}$. This is done in two stages. First, starting from a compact set $K \subset M_{x, y} \times M_{y, z}$, one constructs a so-called pregluing map preg: $K \times\left[\rho(K), \infty\left[\rightarrow \mathcal{P}_{x, z}\right.\right.$ for some $\rho(K)>0$. Here, $\mathcal{P}_{x, z}$ is the manifold of ' $H^{1,2}$-maps' from $\overline{\mathbb{R}}$ to $M$ connecting $x$ to $z$. One then defines a vector bundle $L^{\perp}$ over $K \times\left[\rho(K), \infty\right.$ [ by assigning to $(u, v, \rho) \in K \times\left[\rho(K), \infty\left[\right.\right.$ the $L^{2}$-orthogonal complement $L_{(u, v, \rho)}^{\perp}$ of the image of the tangent map $\left.\mathrm{T} \operatorname{preg}(\rho, \cdot, \cdot)\right|_{(u, v)}$ in $H^{1,2}\left(\operatorname{preg}(u, v, \rho)^{*} \mathrm{~T} M\right)$. One then obtains a true gluing glue $(u, v, \rho)$ of $u$ and $v$ (with the parameter $\rho$ ) as the element of $M_{x, z}$ given by $\exp \circ \xi$ where $\xi=\xi_{(u, v, \rho)}$ is a 'correction term' lying in $L_{(u, v, \rho)}^{\perp}$.

Now to show that $\bigoplus_{\mu \in \mathbb{Z}} \delta_{\mu}$ is a coboundary operator it is essential to have the 'converse gluing theorem', which states that, given a 'broken orbit' $\left(u_{0}, v_{0}\right)$ in the interior of $K$ and an arbitrary sufficiently large gluing parameter $\rho$, every trajectory $w$ in $M_{x, z}$ lying close to $\left(u_{0}, v_{0}\right)$ is contained (up to time-translations) in the range of the gluing map glue $(\cdot, \cdot, \rho)$. In the existing literature (cf. the book of Schwarz [14]) no details of the proof of this fact are found, and it is merely appealed to the fact that $w$ lies $H^{1,2}$-close to 
$\operatorname{preg}\left(u_{0}, v_{0}, \rho\right)$ and to the uniqueness part in the Banach fixed point theorem by which the correction term $\xi_{(u, v, \rho)}$ was obtained. However, this is insufficient since this uniqueness holds only on the vector bundle $L^{\perp}$ (cf. Theorem 1 and the remarks following Lemma 4 below). Therefore we need the additional information that for all $\rho$ large the trajectory $w$ is of the form $w=\exp \circ \zeta$, where $\zeta \in L_{(u, v, \rho)}^{\perp}$ for some $(u, v)$ lying close to $\left(u_{0}, v_{0}\right)$. Moreover, if $w$ is sufficiently close to $\operatorname{preg}\left(u_{0}, v_{0}, \rho\right)$ (in the $H^{1,2}$-sense) we should actually be able to choose $\zeta$ as small as we wish and $(u, v)$ as close to $\left(u_{0}, v_{0}\right)$ as we wish.

In other words, we need to know that there is a $\rho\left(u_{0}, v_{0}\right)$ and for every $\epsilon>0$ there is a $\delta>0$ such that for every $\rho \geq \rho\left(u_{0}, v_{0}\right)$ and every $w \in M_{x, z}$ which lies ' $\delta$-close' to $\operatorname{preg}\left(u_{0}, v_{0}, \rho\right)$ there is a pair $(u, v) \in M_{x, y} \times M_{y, z}$ lying ' $\epsilon$-close' to $\left(u_{0}, v_{0}\right)$ such that $w=\exp \circ \zeta$ with $\zeta \in L_{(u, v, \rho)}^{\perp}$. This means that we need a tubular neighborhood of $\operatorname{preg}\left(u_{0}, v_{0}, \rho\right)$ relative to the bundle $L^{\perp}$ which has thickness $\delta=\delta(\epsilon)$, uniformly in $\rho \geq \rho\left(u_{0}, v_{0}\right)$.

The existence of such a tube cannot be inferred from the usual tubular neighborhood theorem since $\rho$ does not lie in a compact set, and so a different proof is required. Although such a result is probably known to experts we have not found it in the literature.

The purpose of this note is to describe the gluing theorem, to give a precise statement of the above mentioned uniform neighborhood theorem and to show how the latter result can be used to complete the proof of the converse gluing theorem. We merely sketch the arguments here, referring the reader to [9] for details. For simplicity of presentation we work in the setting of the book of Schwarz [14]. However, only minor modifications are needed to obtain analogous results for the various versions of Floer homology developed, e.g., in [6], [7], [8] and [1] as well as for Donaldson's gluing of anti-selfdual connections on four-manifolds (cf. [5]).

2. The gluing theorem. We shall first collect some background material needed for stating the gluing principle and sketching its proof. The reader is referred to [14] for details. Note, however, that our notation differs from that used in [14].

For the time being we consider a fixed (finite or infinite dimensional) smooth Riemannian manifold $M$ modeled on a Hilbert space $E$. Later on we shall impose additional hypotheses on $M$. Let $r(u, v)$ denote the inner product of $u$ and $v$, where $u,\left.v \in \mathrm{T} M\right|_{x}$ for some $x \in M$. Let $\tau: \mathrm{T} M \rightarrow M$ denote the projection map, K: TT $M \rightarrow \mathrm{T} M$ be the Levi-Civita connection map defined by the Riemannian structure $r(\cdot, \cdot)$ and exp: $\mathcal{O} \subset$ $\mathrm{T} M \rightarrow M$ be the corresponding exponential map.

There are sets $\mathcal{D} \subset \mathcal{O}$ and $\mathcal{C} \subset M \times M$ such that $\mathcal{D}$ is an open neighborhood of the zero section in $\mathrm{T} M, \mathcal{C}$ is an open neighborhood of the diagonal in $M \times M$ and the map $(\tau, \exp )$ is a diffeomorphism of $\mathcal{D}$ onto $\mathcal{C}$. We denote by $\Lambda: \mathcal{C} \rightarrow \mathcal{D}$ the inverse map of $(\tau, \exp )$.

For $x \in M, v \in W:=\left.\mathcal{O} \cap \mathrm{T} M\right|_{x}$ and $\left.w \in \mathrm{T} M\right|_{x}$ set

$$
\nabla_{2} \exp (v) w:=\operatorname{Texp}_{x}(v, w) .
$$

Here, $\exp _{x}$ is the restriction of $\exp$ to $W$. Since $W$ is open in the (Hilbertable) space $\left.\mathrm{T} M\right|_{x}$, we can identify $\mathrm{T} W$ with $W \times\left.\mathrm{T} M\right|_{x}$. Note that this defines a bounded linear $\operatorname{map} \nabla_{2} \exp (v):\left.\left.\mathrm{T} M\right|_{x} \rightarrow \mathrm{T} M\right|_{\exp (v)}$. 
Let $\overline{\mathbb{R}}:=[-\infty,+\infty]$. Following [14] we define the map

$$
\bar{h}: \overline{\mathbb{R}} \rightarrow[-1,1]
$$

as

$$
\bar{h}(t)= \begin{cases}t\left(1+t^{2}\right)^{-1 / 2} & \text { for } t \in \mathbb{R} \\ \pm 1 & \text { for } t= \pm \infty .\end{cases}
$$

Using the singleton atlas $\{\bar{h}\}$ we view $\overline{\mathbb{R}}$ as a smooth manifold (with boundary).

Given any map $u: \overline{\mathbb{R}} \rightarrow M$ and $\mathcal{V} \subset \mathrm{T} M$ we set

$$
u^{*} \mathcal{V}=\left\{(t, v) \mid t \in \overline{\mathbb{R}} \text { and }\left.v \in \mathrm{T} M\right|_{u(t)} \cap \mathcal{V}\right\}
$$

It is clear that $u^{*} \mathcal{V} \subset u^{*} \mathrm{~T} M$.

A section $\xi$ of $u^{*} \mathcal{V}$ is a map defined on $\overline{\mathbb{R}}$ such that $\xi(t)=\left(t, \xi^{p}(t)\right)$ where $\xi^{p}(t) \in$ $\left.\mathrm{T} M\right|_{u(t)} \cap \mathcal{V}$ for all $t \in \overline{\mathbb{R}}$. $\xi^{p}$ is called the principal part of $\xi$.

Now assume that $u: \overline{\mathbb{R}} \rightarrow M$ is smooth. Then $u^{*} \mathrm{~T} M$ is a smooth vector bundle over $\overline{\mathbb{R}}$ so it has a smooth trivialization $\phi: u^{*} \mathrm{~T} M \rightarrow \overline{\mathbb{R}} \times E$. A trivialization of a section $\xi$ of $u^{*} \mathcal{V}$ (with respect to $\phi$ ) is the map $\xi_{\phi}: \overline{\mathbb{R}} \rightarrow E$ such that $\left(t, \xi_{\phi}(t)\right)=\phi(\xi(t))$ for all $t \in \overline{\mathbb{R}}$.

We define $H^{1,2}\left(u^{*} \mathcal{V}\right)$ to be the set of all sections $\xi$ of $u^{*} \mathcal{V}$ such that $\xi_{\phi}: \overline{\mathbb{R}} \rightarrow E$ is continuous and $\xi_{\phi} \in H^{1,2}(\mathbb{R}, E)$. We also define $L^{2}\left(u^{*} \mathcal{V}\right)$ to be the set of all sections $\xi$ of $u^{*} \mathcal{V}$ such that $\xi_{\phi} \in L^{2}(\mathbb{R}, E)$. Using the differentiable structure on $\overline{\mathbb{R}}$ defined above it is shown that these sets are independent of the choice of $\phi$.

The linear and the topological structures on $H^{1,2}\left(u^{*} \mathrm{~T} M\right)$ and $L^{2}\left(u^{*} \mathrm{~T} M\right)$ are defined in an obvious way using the trivialization $\phi$. Again these structures are independent of $\phi$. If $\mathcal{V}$ is open in TM then $H^{1,2}\left(u^{*} \mathcal{V}\right)$ is open in $H^{1,2}\left(u^{*} \mathrm{~T} M\right)$.

If $\xi: \overline{\mathbb{R}} \rightarrow \mathrm{T} M$ lies in $H^{1,2}\left(u^{*} \mathrm{~T} M\right)$ then $\xi$ is differentiable at almost every $t \in \mathbb{R}$. For such $t$ we have $\mathrm{T} \xi(t, 1) \in \mathrm{TT} M$ and we set $\nabla \xi(t):=\mathrm{K}(\mathrm{T} \xi(t, 1)) \in \mathrm{T} M$.

If $\xi$ and $\eta \in H^{1,2}\left(u^{*} \mathrm{~T} M\right)$ then we set

$$
\langle\xi, \eta\rangle_{1,2}:=\int_{\mathbb{R}} r(\xi(t), \eta(t)) \mathrm{d} t+\int_{\mathbb{R}} r(\nabla \xi(t), \nabla \eta(t)) \mathrm{d} t .
$$

This defines a complete inner product on the space $H^{1,2}\left(u^{*} \mathrm{~T} M\right)$.

Similarly, if $\xi$ and $\eta \in L^{2}\left(u^{*} \mathrm{~T} M\right)$ then we set

$$
\langle\xi, \eta\rangle_{0,2}:=\int_{\mathbb{R}} r(\xi(t), \eta(t)) \mathrm{d} t,
$$

thus defining a complete inner product on $L^{2}\left(u^{*} \mathrm{~T} M\right)$.

For every trivialization $\phi$ of $u^{*} \mathrm{~T} M$ the map

$$
H^{1,2}\left(u^{*} \mathrm{~T} M\right) \rightarrow H^{1,2}(\mathbb{R}, E) \text { defined by } \xi \mapsto \xi_{\phi}
$$

and the map

$$
L^{2}\left(u^{*} \mathrm{~T} M\right) \rightarrow L^{2}(\mathbb{R}, E) \text { defined by } \xi \mapsto \xi_{\phi}
$$

are Hilbert space isomorphisms.

Given points $x, y \in M$ we define $\mathcal{P}_{x, y}$ to be the set of all functions $u: \overline{\mathbb{R}} \rightarrow M$ for which there is a smooth map $h: \overline{\mathbb{R}} \rightarrow M$ such that $h(-\infty)=x, h(\infty)=y,(h(t), u(t)) \in \mathcal{C}$ for $t \in \overline{\mathbb{R}}$, and such that $\xi \in H^{1,2}\left(h^{*} \mathrm{~T} M\right)$, where $\xi$ is the section of $h^{*} \mathrm{~T} M$ with principal part defined on $\overline{\mathbb{R}}$ by $\xi^{p}(t)=\Lambda(h(t), u(t))$. In other words, $u \in \mathcal{P}_{x, y}$ if and only if there is a 
smooth map $h: \overline{\mathbb{R}} \rightarrow M$ with $h(-\infty)=x, h(\infty)=y$ and there is a section $\xi \in H^{1,2}\left(h^{*} \mathcal{D}\right)$ such that $u(t)=\exp \left(\xi^{p}(t)\right)$ for $t \in \overline{\mathbb{R}}$. In this case we say that $u$ is $H^{1,2}$-carried by $h$.

The map

$$
A_{h}: H^{1,2}\left(h^{*} \mathcal{D}\right) \rightarrow \mathcal{P}_{x, y}, \quad \xi \mapsto u
$$

with $u(t)=\exp \left(\xi^{p}(t)\right)$ for $t \in \overline{\mathbb{R}}$, is well-defined and bijective onto its range. The set of the inverses $A_{h}^{-1}$, where $h: \overline{\mathbb{R}} \rightarrow M$ is arbitrary smooth satisfying $h(-\infty)=x, h(\infty)=y$, is a smooth atlas which defines the structure of a differentiable manifold on $\mathcal{P}_{x, y}$.

For an arbitrary $u \in \mathcal{P}_{x, y}$ define the $H^{1,2}$-fibre $H^{1,2}\left(u^{*} \mathrm{~T} M\right)$ (resp. the $L^{2}$-fibre $\left.L^{2}\left(u^{*} \mathrm{~T} M\right)\right)$ at $u$ as follows. Take an arbitrary smooth map $h$ such that $u$ is $H^{1,2}$-carried by $h$. If $\xi \in H^{1,2}\left(h^{*} \mathcal{D}\right)$ is such that $u(t)=\exp \left(\xi^{p}(t)\right)$ for $t \in \overline{\mathbb{R}}$, then let $H^{1,2}\left(u^{*} T M\right)$ (resp. $\left.L^{2}\left(u^{*} \mathrm{~T} M\right)\right)$ be the set of all sections $\beta$ of $u^{*} \mathrm{~T} M$ such that for some $\alpha \in H^{1,2}\left(h^{*} \mathrm{~T} M\right)$, (resp. for some $\alpha \in L^{2}\left(h^{*} \mathrm{~T} M\right)$ )

$$
\beta^{p}(t)=\nabla_{2} \exp \left(\xi^{p}(t)\right) \cdot \alpha^{p}(t), \quad t \in \overline{\mathbb{R}} .
$$

The definition of $H^{1,2}\left(u^{*} \mathrm{~T} M\right)$ (resp. $L^{2}\left(u^{*} \mathrm{~T} M\right)$ ) is independent of the choice of $h$ and for $u$ smooth the new definition of $H^{1,2}\left(u^{*} \mathrm{~T} M\right)$ coincides with the old one. We define $H^{1,2}\left(\mathcal{P}_{x, y}^{*} \mathrm{~T} M\right)\left(\right.$ resp. $\left.L^{2}\left(\mathcal{P}_{x, y}^{*} \mathrm{~T} M\right)\right)$ to be the disjoint union of the fibres $H^{1,2}\left(u^{*} \mathrm{~T} M\right)$ (resp. $\left.L^{2}\left(u^{*} \mathrm{~T} M\right)\right)$, for all $u \in \mathcal{P}_{x, y}$.

The map

$$
B_{h}: H^{1,2}\left(h^{*} \mathcal{D}\right) \times H^{1,2}\left(h^{*} \mathrm{~T} M\right) \rightarrow H^{1,2}\left(\mathcal{P}_{x, y}^{*} \mathrm{~T} M\right), \quad(\xi, \alpha) \mapsto \beta
$$

(resp. the map

$$
\left.B_{h}: H^{1,2}\left(h^{*} \mathcal{D}\right) \times L^{2}\left(h^{*} \mathrm{~T} M\right) \rightarrow L^{2}\left(\mathcal{P}_{x, y}^{*} \mathrm{~T} M\right), \quad(\xi, \alpha) \mapsto \beta,\right)
$$

where

$$
\beta^{p}(t)=\nabla_{2} \exp \left(\xi^{p}(t)\right) \cdot \alpha^{p}(t), \quad t \in \overline{\mathbb{R}},
$$

is bijective onto its range. The set of the inverses of all these maps (as $h$ is varied) forms a smooth vector bundle atlas on $H^{1,2}\left(\mathcal{P}_{x, y}^{*} \mathrm{~T} M\right)$ (resp. on $\left.L^{2}\left(\mathcal{P}_{x, y}^{*} \mathrm{~T} M\right)\right)$.

The vector bundle $H^{1,2}\left(\mathcal{P}_{x, y}^{*} \mathrm{~T} M\right)$ is isomorphic to and can be identified with the tangent bundle of $\mathcal{P}_{x, y}$.

We shall now define the pregluing operation. Let $\beta^{-}: \overline{\mathbb{R}} \rightarrow \mathbb{R}$ and $\beta^{+}: \overline{\mathbb{R}} \rightarrow \mathbb{R}$ be two smooth functions such that

$$
\beta^{-} \equiv 1 \text { on }[-\infty,-1] \text { and } \beta^{-} \equiv 0 \text { on }[0, \infty]
$$

while

$$
\beta^{+} \equiv 1 \text { on }[1, \infty] \text { and } \beta^{+} \equiv 0 \text { on }[-\infty, 0] .
$$

Let $y \in M$ be arbitrary. Given two maps $u, v: \overline{\mathbb{R}} \rightarrow M$ and a real number $\rho$ we say that $\operatorname{preg}(u, v, \rho)$ is defined if

$$
(y, u(t+\rho)) \in \mathcal{C} \quad \text { and } \beta^{-}(t) \cdot \Lambda(y, u(t+\rho)) \in \mathcal{D} \text { for } t \in[-1,0]
$$

and

$$
(y, v(t-\rho)) \in \mathcal{C} \quad \text { and } \beta^{+}(t) \cdot \Lambda(y, v(t-\rho)) \in \mathcal{D} \text { for } t \in[0,1] .
$$

Here, '.' is just multiplication with scalars, which is well-defined since, e.g., $\Lambda(y, u(t+\rho)) \in$ $\left.\mathrm{T} M\right|_{y}$. 
If $\operatorname{preg}(u, v, \rho)$ is defined then we set

$$
\operatorname{preg}(u, v, \rho)(t)= \begin{cases}u(t+\rho) & \text { for } t \in[-\infty,-1], \\ \exp \left(\beta^{-}(t) \cdot \Lambda(y, u(t+\rho))\right) & \text { for } t \in[-1,0], \\ \exp \left(\beta^{+}(t) \cdot \Lambda(y, v(t-\rho))\right) & \text { for } t \in[0,1] \\ v(t-\rho) & \text { for } t \in[1, \infty]\end{cases}
$$

It follows that $\operatorname{preg}(u, v, \rho)$ is a map defined on $\overline{\mathbb{R}}$ with values in $M$.

Now let $f: M \rightarrow \mathbb{R}$ be a smooth function and define the gradient $\nabla f$ of $f$ as the vector field on $M$ such that

$$
(f(x), r(\nabla f(x), v))=\mathrm{T} f(v) \text { for all } x \in M \text { and }\left.v \in \mathrm{T} M\right|_{x} .
$$

Here, as usual, we identify $T \mathbb{R}$ with $\mathbb{R} \times \mathbb{R}$. Let $\pi$ be the (local) flow defined by the solutions of the differential equation

$$
\dot{u}=-\nabla f \circ u \text {. }
$$

$\pi$ is called the negative gradient flow of $f$. A point $x \in M$ is an equilibrium of $\pi$ if and only if $x$ is a critical point of $f$, i.e. $X(x)=0_{x}$, where $X=\nabla f$. Here, the notation $0_{x}$ is used to denote the zero vector in the tangent space $\left.\mathrm{T} M\right|_{x}$. A critical point $x$ of $f$ is called hyperbolic if and only if for some (and hence every) chart $\phi$ at $x$ the differential

$$
\mathrm{D}\left(\mathrm{T} \phi \circ X \circ \phi^{-1}\right)(\phi(x)): E \rightarrow E
$$

does not have zero in its spectrum.

Let $x$ and $y$ be two critical points of $f$. Define the map

$$
F_{x, y}: \mathcal{P}_{x, y} \rightarrow L^{2}\left(\mathcal{P}_{x, y}^{*} \mathrm{~T} M\right) \text { by } u \mapsto \dot{u}+\nabla f \circ u .
$$

The map $F_{x, y}$ is a well-defined smooth section of the vector bundle $L^{2}\left(\mathcal{P}_{x, y}^{*} \mathrm{~T} M\right)$. Let $M_{x, y}$ be the set of the zeros of this section, i.e. $u \in M_{x, y}$ if and only if $F_{x, y}(u)=0_{u}$. It follows from the usual regularity theory that $u \in M_{x, y}$ if and only if $\left.u\right|_{\mathbb{R}}$ is a (smooth) solution of the flow $\pi$ on $\mathbb{R}$ such that $u(t) \rightarrow x$ as $t \rightarrow-\infty$ and $u(t) \rightarrow y$ as $t \rightarrow \infty$. Such a solution is also called a heteroclinic solution, or an instanton, from $x$ to $y$.

If the tangent map $\left.\mathrm{T} F_{x, y}\right|_{u}$ is a surjective Fredholm operator at every $u \in M_{x, y}$, then $M_{x, y}$ is a finite dimensional smooth submanifold of $\mathcal{P}_{x, y}$ with

$$
\left.\mathrm{T} M_{x, y}\right|_{u}=\left.\operatorname{ker} \mathrm{T} F_{x, y}\right|_{u} \text { for every } u \in M_{x, y} \text {. }
$$

Now let $x, y$ and $z$ be three hyperbolic critical points of $f$. Let $K$ be a compact set in $M_{x, y} \times M_{y, z}$. Suppose that for every $(u, v) \in K$ the tangent maps $\left.\mathrm{T} F_{x, y}\right|_{u}$ and $\left.\mathrm{T} F_{y, z}\right|_{v}$ are surjective Fredholm operators. Then there is a $\rho(K)>0$ and an open neighborhood $U(K)$ of $K$ in $M_{x, y} \times M_{y, z}$ such that $\operatorname{preg}(u, v, \rho)$ is defined for all $(u, v) \in U(K)$ and all $\rho \in[\rho(K), \infty[$. Moreover, $\operatorname{preg}(u, v, \rho): \overline{\mathbb{R}} \rightarrow M$ is a smooth function of $t \in \overline{\mathbb{R}}$. Thus we obtain the induced map

$$
\text { preg: } U(K) \times\left[\rho(K), \infty\left[\rightarrow \mathcal{P}_{x, z},(u, v, \rho) \mapsto \operatorname{preg}(u, v, \rho) .\right.\right.
$$

This map is smooth so for every $\rho \in[\rho(K), \infty[$ the partial map

$$
\operatorname{preg}_{\rho}: U(K) \rightarrow \mathcal{P}_{x, z},(u, v) \mapsto \operatorname{preg}(u, v, \rho),
$$


is smooth as well. Therefore, for every $(u, v) \in U(K)$ there is a well-defined tangent map

$$
\left.\operatorname{T}_{\operatorname{preg}}\right|_{(u, v)}:\left.\mathrm{T} M_{x, y}\right|_{u} \times\left.\left.\mathrm{T} M_{y, z}\right|_{v} \rightarrow \mathrm{TP} \mathcal{P}_{x, y}\right|_{w}=H^{1,2}\left(w^{*} \mathrm{~T} M\right),
$$

where $w:=\operatorname{preg}_{\rho}(u, v)$.

Define

$L_{(u, v, \rho)}^{\perp}:=$

$$
\left\{\xi \in H^{1,2}\left(w^{*} \mathrm{~T} M\right) \mid\left\langle\operatorname{T}_{\operatorname{preg}_{\rho}}(a, b), \xi\right\rangle_{0,2}=0 \text { for all }\left.(a, b) \in \mathrm{T} M_{x, y}\right|_{u} \times\left.\mathrm{T} M_{y, z}\right|_{v}\right\} .
$$

In other words, $\xi$ lies in $L_{(u, v, \rho)}^{\perp}$ if and only if $\xi \in H^{1,2}\left(w^{*} \mathrm{~T} M\right)$ and $\xi$ is $\langle\cdot, \cdot\rangle_{0,2}$-orthogonal to the image of the tangent map T $\left.\operatorname{preg}_{\rho}\right|_{(u, v)}$. The set $L_{(u, v, \rho)}^{\perp}$ is a closed linear subspace of $H^{1,2}\left(w^{*} \mathrm{~T} M\right)$.

The importance of $L_{(u, v, \rho)}^{\perp}$ lies in the following result, whose intuitive content is the following: one would like to 'glue' the instanton $u$ from $x$ to $y$ with the instanton $v$ from $y$ to $z$ in such a way as to obtain an instanton from $x$ to $z$. Now the result $w=\operatorname{preg}_{\rho}(u, v)$ of the pregluing operation lies in $\mathcal{P}_{x, z}$ but may fail to be an instanton, i.e. a solution of negative gradient flow $\pi$ of $f$. However, by perturbing $w$ with a small correction term $\xi$ lying in $L_{(u, v, \rho)}^{\perp}$ one does obtain an instanton $\tilde{w}$ of the form $\tilde{w}=\exp \circ \xi^{p}$, i.e.

$$
\tilde{w}(t)=\exp \left(\xi^{p}(t)\right), \quad t \in \overline{\mathbb{R}} .
$$

From now on we assume that the manifold $M$ is finite-dimensional and complete.

TheOREm 1 (Gluing principle, [14]). The number $\rho(K)$, the open neighborhood $U(K)$ of $K$ and a number $\epsilon(K)>0$ can be chosen in such a way that for every $\rho \in[\rho(K), \infty[$ and every $(u, v) \in U(K)$ there is a unique $\xi=\xi_{(u, v, \rho)}$ such that

1) $\xi \in L_{(u, v, \rho)}^{\perp}$,

2) $\|\xi\|_{1,2}<\epsilon(K)$, and

3) $F_{x, z}(\tilde{w})=0_{\tilde{w}}$ where $\tilde{w}=\exp \circ \xi^{p}$.

Furthermore, the map

$$
\Gamma: U(K) \times\left[\rho(K), \infty\left[\rightarrow H^{1,2}\left(\mathcal{P}_{x, z}^{*} \mathrm{~T} M\right), \quad(u, v, \rho) \mapsto \xi_{(u, v, \rho)}\right.\right.
$$

is smooth.

Finally, there are positive real constants $M(K)$ and $m(K)$ such that

$$
\left\|\xi_{(u, v, \rho)}\right\|_{1,2} \leq M(K) e^{-m(K) \rho}, \quad(u, v, \rho) \in U(K) \times[\rho(K), \infty[.
$$

Using Theorem 1 we thus obtain the well-defined gluing map

$$
\text { glue: } U(K) \times\left[\rho(K), \infty\left[\rightarrow M_{x, z}, \quad(u, v, \rho) \mapsto \exp \circ \xi^{p} \text { where } \xi=\xi_{(u, v, \rho)} .\right.\right.
$$

The gluing map is smooth.

We shall now indicate indicate the main steps in the proof of Theorem 1. For $\chi=$ $(u, v, \rho) \in U(K) \times\left[\rho(K), \infty\left[\right.\right.$ let $w=\operatorname{preg}(u, v, \rho)$. Since $w$ is a smooth function in $\mathcal{P}_{x, z}$ we can consider the coordinate expression $F_{\chi}$ of $F=F_{x, z}$ in the charts $A_{w}$ and $B_{w}$ :

$$
F_{\chi}=\operatorname{Pr}_{2} \circ B_{w}^{-1} \circ F \circ A_{w}: \operatorname{dom} F_{\chi} \subset H^{1,2}\left(w^{*} \mathrm{~T} M\right) \rightarrow L^{2}\left(w^{*} \mathrm{~T} M\right) .
$$

Here

$$
\operatorname{Pr}_{2}: H^{1,2}\left(w^{*} \mathcal{D}\right) \times L^{2}\left(w^{*} \mathrm{~T} M\right) \rightarrow L^{2}\left(w^{*} \mathrm{~T} M\right)
$$

is the projection onto the second factor. The following result holds: 
Lemma 1 ([14, Proposition 2.50]). Given $\chi, w$ and $F_{\chi}$ as above we have $F(w)=F_{\chi}(0)$, where 0 is the zero in $H^{1,2}\left(w^{*} \mathrm{~T} M\right)$. The set $\operatorname{dom} F_{\chi}$ is open in $H^{1,2}\left(w^{*} \mathrm{~T} M\right)$.

Moreover, the number $\rho(K)$ and the open neighborhood $U(K)$ of $K$ can be chosen in such a way that for every $\chi=(u, v, \rho) \in U(K) \times[\rho(K), \infty[$ the map

$$
\mathrm{D} F_{\chi}(0): H^{1,2}\left(w^{*} \mathrm{~T} M\right) \rightarrow L^{2}\left(w^{*} \mathrm{~T} M\right)
$$

is surjective and the map

$$
P_{\chi} \circ \operatorname{T}_{p^{\prime} e g}(u, v):\left.\mathrm{T}_{x, y}\right|_{u} \times\left.\mathrm{T}_{y, z}\right|_{v} \rightarrow \operatorname{ker} \mathrm{D} F_{\chi}(0)
$$

is bijective, where $w=\operatorname{preg}(u, v, \rho)$ and

$$
P_{\chi}: H^{1,2}\left(w^{*} \mathrm{~T} M\right) \rightarrow \operatorname{ker} \mathrm{D} F_{\chi}(0)
$$

is the $\langle\cdot, \cdot\rangle_{0,2}$-orthogonal projection of $H^{1,2}\left(w^{*} \mathrm{~T} M\right)$ onto $\operatorname{ker} \mathrm{D} F_{\chi}(0)$.

Let $U(K)$ and $\rho(K)$ be as in Lemma 1. Given $\chi=(u, v, \rho) \in U(K) \times[\rho(K), \infty[$ set $w=\operatorname{preg}(u, v, \rho)$. Lemma 1 and obvious dimension arguments imply that

$$
H^{1,2}\left(w^{*} \mathrm{~T} M\right)=\operatorname{ker} \mathrm{D} F_{\chi}(0) \oplus L_{\chi}^{\perp} .
$$

Hence

$$
\left.\mathrm{D} F_{\chi}(0)\right|_{L_{\chi}^{\perp}}: L_{\chi}^{\perp} \rightarrow L^{2}\left(w^{*} \mathrm{~T} M\right)
$$

is bijective and we define the map

$$
G_{\chi}: L^{2}\left(w^{*} \mathrm{~T} M\right) \rightarrow L_{\chi}^{\perp}
$$

to be the inverse of $\left.\mathrm{D} F_{\chi}(0)\right|_{L_{\chi}}$.

LEMma 2 ([14, Lemma 2.51]). The number $\rho(K)$ and the open neighborhood $U(K)$ of $K$ can be chosen in such a way that for some positive real constant $C_{1}(K)$ and for every $\chi=(u, v, \rho) \in U(K) \times[\rho(K), \infty[$

$\left\|G_{\chi} \eta\right\|_{1,2} \leq C_{1}(K)\|\eta\|_{0,2} \quad$ for all $\eta \in L^{2}\left(w^{*} \mathrm{~T} M\right)$, where $w=\operatorname{preg}(u, v, \rho)$.

Next, with our usual notation, define the map

$$
N_{\chi}: \operatorname{dom} F_{\chi} \rightarrow L^{2}\left(w^{*} \mathrm{~T} M\right)
$$

by

$$
N_{\chi}(\xi)=F_{\chi}(\xi)-F_{\chi}(0)-\mathrm{D} F_{\chi}(0) \xi \quad \text { for all } \xi \in \operatorname{dom} F_{\chi}
$$

Lemma 3 ([14, p. 86]). The number $\rho(K)$ and the open neighborhood $U(K)$ of $K$ can be chosen in such a way that for some positive real constants $C_{2}(K), M(K), m(K), \delta(K)$ and for every $\chi=(u, v, \rho) \in U(K) \times[\rho(K), \infty[$

$$
\begin{gathered}
B_{\delta(K)}:=\left\{\xi \in H^{1,2}\left(w^{*} \mathrm{~T} M\right) \mid\|\xi\|_{1,2} \leq \delta(K)\right\} \subset \operatorname{dom} F_{\chi}, \\
\left\|F_{\chi}(0)\right\|_{0,2} \leq M(K) e^{-m(K) \rho}
\end{gathered}
$$

and

$$
\left\|N_{\chi}\left(\xi_{1}\right)-N_{\chi}\left(\xi_{2}\right)\right\|_{0,2} \leq C_{2}(K)\left(\left\|\xi_{1}\right\|_{1,2}+\left\|\xi_{2}\right\|_{1,2}\right)\left\|\xi_{1}-\xi_{2}\right\|_{1,2}
$$

for all $\xi_{1}, \xi_{2} \in B_{\delta(K)}$, where $w=\operatorname{preg}(u, v, \rho)$. 
LEMma 4 (cf. [14, Lemma 2.52]). Suppose that $X$ and $Y$ are Banach spaces, $\mathcal{E}$ is an open neighborhood of 0 in $X$ and $g: \mathcal{E} \rightarrow Y, N: \mathcal{E} \rightarrow Y, S: X \rightarrow Y$ and $G: Y \rightarrow X$ are maps such that $S$ and $G$ are linear,

$$
\begin{gathered}
S \circ G=\operatorname{id}_{Y}, \\
g(a)=g(0)+S a+N(a) \quad \text { for all } a \in \mathcal{E},
\end{gathered}
$$

and there is a positive real constant $C$ such that

$$
B_{\epsilon} \subset \mathcal{E}, \quad\|G g(0)\| \leq \epsilon / 2
$$

and

$$
\|G N(a)-G N(b)\| \leq C(\|a\|+\|b\|)\|a-b\|
$$

for all $a, b \in X$ with $\|a\|,\|b\| \leq \epsilon$, where $\epsilon:=1 /(5 C)$ and $B_{\epsilon}$ is the closed $\epsilon$-ball in $X$ centered at zero.

Then there exists a unique $a_{0} \in X$ such that

1) $a_{0} \in G(Y)$,

2) $a_{0} \in B_{\epsilon}$,

3) $g\left(a_{0}\right)=0$.

In addition we have $\left\|a_{0}\right\|<\epsilon$ and $\left\|a_{0}\right\| \leq 2\|G g(0)\|$.

The proof of Lemma 4 is obtained by showing that the zeros of $g$ lying in $G(Y) \cap B_{\epsilon}$ are exactly the fixed points of the map

$$
\Phi(x):=-G(g(0)+N(x)), \quad x \in B_{\epsilon}
$$

and proving that $\Phi$ is a $q$-contraction (with $q=1 / 2$ ) and maps $B_{\epsilon}$ into itself. Note that there may be other zeros of $g$ in $B_{\epsilon}$ which do not lie in $G(Y)$. In fact, the totality of all zeros of $g$ in $B_{\epsilon}$ is a manifold of dimension equal to the dimension of the kernel of $S$.

We can now give a proof of Theorem 1 as follows: Define

$$
C(K):=\max \left(C_{1}(K) C_{2}(K), 1 /(5 \delta(K))\right) \text { and } \epsilon(K):=1 /(5 C(K)) .
$$

Choose $U(K)$ and $\rho(K)>0$ such that the assertions Lemmas 1 - 3 hold and such that

$$
C_{1}(K) M(K) e^{-m(K) \rho(K)} \leq \epsilon(K) / 2 .
$$

For every

$$
\chi=(u, v, \rho) \in U(K) \times[\rho(K), \infty[\text { and } w=\operatorname{preg}(u, v, \rho)
$$

the assumptions of Lemma 4 are satisfied with $X=H^{1,2}\left(w^{*} \mathrm{~T} M\right), Y=L^{2}\left(w^{*} \mathrm{~T} M\right)$, $g=F_{\chi}, N=N_{\chi}, S=\mathrm{DF}_{\chi}(0), G=G_{\chi}$ and $C=C(K)$. Letting $\xi_{(u, v, \rho)}:=a_{0}$ where $a_{0}$ is as in the statement of Lemma 4, we obtain all assertions of Theorem 1 except for the smoothness of the map $\Gamma$. To prove that $\Gamma$ is smooth one first uses the fact, following from Lemma 1, that the union of all the spaces $L_{\chi}^{\perp}$ is a vector bundle over $U(K) \times\left[\rho(K), \infty\left[\right.\right.$. Then one can write equation $F_{\chi}(\xi)=0$ in coordinates around any fixed $\chi_{0}=\left(u_{0}, v_{0}, \rho_{0}\right)$. One then obtains an abstract equation of the form $g(\chi, a)=0$, with $\chi$ lying in a neighborhood $W$ of $\chi_{0}$ where $g: W \times X \rightarrow Y$ is smooth with $X=H^{1,2}\left(w_{0}^{*} \mathrm{~T} M\right)$, $Y=L^{2}\left(w_{0}^{*} \mathrm{~T} M\right)$ and $w_{0}:=\operatorname{preg}\left(u_{0}, v_{0}, \rho_{0}\right)$. Then the implicit function theorem (or a version of Lemma 4 with parameters) implies the smoothness of $\Gamma$. 
3. The converse gluing principle and a uniform tubular neighborhood theorem. The gluing map has an important imbedding property ([14, Proposition 2.56]), which we do not discuss here. Instead, we shall concentrate on the asymptotic behavior of the gluing map and the converse gluing theorem. For this we need a few definitions:

Given a map $u: \mathbb{R} \rightarrow M$ and a real number $\tau$ the map $u(\cdot+\tau): \mathbb{R} \rightarrow M$ defined by $t \mapsto u(t+\tau)$ is called the $\tau$-translate of $u$.

Let $u_{n}: \mathbb{R} \rightarrow M, n \in \mathbb{N}$, be a sequence of continuous maps. We say that $u_{n}$ converges almost uniformly to the continuous map $v: \mathbb{R} \rightarrow M$ if and only if for every positive real number $R$

$$
\sup _{t \in[-R, R]} d\left(u_{n}(t), v(t)\right) \rightarrow 0 \quad \text { as } n \rightarrow \infty .
$$

Here, $d$ is the Riemannian distance on $M$.

Let $w_{n}: \mathbb{R} \rightarrow M, n \in \mathbb{N}$, be a sequence of continuous maps. We say that $w_{n}$ converges almost uniformly to the pair $(u, v)$ of continuous maps $u: \mathbb{R} \rightarrow M$ and $v: \mathbb{R} \rightarrow M$ if and only if there are sequences $\sigma_{n}$ and $\tau_{n}$ of real numbers such that the $\sigma_{n}$-translate sequence $w\left(\cdot+\sigma_{n}\right), n \in \mathbb{N}$, converges almost uniformly to $u$ and the $\tau_{n}$-translate sequence $w\left(\cdot+\tau_{n}\right)$, $n \in \mathbb{N}$, converges almost uniformly to $v$.

We have the following

THEOREM 2 (Limit behavior of the gluing map, cf. [14]). If $\left(u_{n}, v_{n}\right), n \in \mathbb{N}$, is a sequence in $K$ such that for some $\left(u_{0}, v_{0}\right) \in K, u_{n}$ converges almost uniformly to $u_{0}$ and $v_{n}$ converges almost uniformly to $v_{0}$, and if $\rho_{n} \rightarrow \infty$, then glue $\left(u_{n}, v_{n}, \rho_{n}\right)$ converges almost uniformly to the pair $\left(u_{0}, v_{0}\right)$.

Theorem 2 admits a converse result:

THEOREM 3 (Converse gluing theorem). If $\left(u_{0}, v_{0}\right)$ lies in the interior of $K$, if $w_{n}$, $n \in \mathbb{N}$, is a sequence in $M_{x, z}$ which converges almost uniformly to the pair $\left(u_{0}, v_{0}\right)$ and if $\rho_{n} \rightarrow \infty$ then there is a sequence $\left(u_{n}, v_{n}\right), n \in \mathbb{N}$, in $K$ such that $u_{n}$ converges almost uniformly to $u_{0}, v_{n}$ converges almost uniformly to $v_{0}$, there is a sequence $\tau_{n}, n \in \mathbb{N}$, of real numbers and there is an $n_{1}$ such that

$$
w_{n}\left(\cdot+\tau_{n}\right)=\operatorname{glue}\left(u_{n}, v_{n}, \rho_{n}\right) \quad \text { for all } n \geq n_{1} .
$$

Theorem 3 plays a crucial role in the proof of the fact that the operator $\delta_{\mu}$ defined in the Introduction is a coboundary operator.

The following result is the main step in the proof of the converse gluing theorem:

THEOREM 4 (Uniform tubular neighborhood theorem, [9]). Let $\left(u_{0}, v_{0}\right) \in M_{x, y} \times M_{y, z}$ be arbitrary. Then there is an open neighborhood $U=U\left(u_{0}, v_{0}\right)$ of $\left(u_{0}, v_{0}\right)$ in $M_{x, y} \times M_{y, z}$ and there is a $\rho\left(u_{0}, v_{0}\right)>0$ such that for every $\epsilon>0$ there is a $\delta=\delta\left(u_{0}, v_{0}, \epsilon\right)>0$ with the following property:

Whenever $(\bar{u}, \bar{v}) \in U, \rho \in\left[\rho\left(u_{0}, v_{0}\right), \infty\left[, \bar{w}:=\operatorname{preg}(\bar{u}, \bar{v}, \rho)\right.\right.$ and $w \in \mathcal{P}_{x, z}$ is such that

$$
w=\exp \circ \bar{\zeta}^{p} \text { for some } \bar{\zeta} \in H^{1,2}\left(\bar{w}^{*} \mathcal{D}\right) \text { with }\|\bar{\zeta}\|_{1,2}<\delta \text {, }
$$

then there is $a(u, v) \in M_{x, y} \times M_{y, z}$ such that

$$
u=\exp \circ \xi^{p} \text { for some } \xi \in H^{1,2}\left(\bar{u}^{*} \mathcal{D}\right) \text { with }\|\xi\|_{1,2}<\epsilon,
$$




$$
v=\exp \circ \eta^{p} \text { for some } \eta \in H^{1,2}\left(\bar{v}^{*} \mathcal{D}\right) \text { with }\|\eta\|_{1,2}<\epsilon
$$

and

$$
w=\exp \circ \zeta^{p} \text { for some } \zeta \in H^{1,2}\left(\operatorname{preg}(u, v, \rho)^{*} \mathcal{D}\right) \text { with }\|\zeta\|_{1,2}<\epsilon
$$

and such that

$$
\zeta \in L_{(u, v, \rho)}^{\perp} .
$$

We shall also need the following known result, which was proved in [6] in the context of Floer homology:

Lemma 5 (cf. [6, pp. 532-533]). Under the assumptions of Theorem 3 let $\bar{n} \in \mathbb{N}$ be such that $\rho_{n} \geq \rho(K)$ for $n \geq \bar{n}$. Set

$$
b_{n}:=\operatorname{preg}\left(u_{0}, v_{0}, \rho_{n}\right), \quad n \geq \bar{n} .
$$

There are sequences $\tau_{n} \in \mathbb{R}$ and $\bar{\zeta}_{n} \in H^{1,2}\left(b_{n}^{*} \mathcal{D}\right), n \geq \bar{n}$, with $\left\|\bar{\zeta}_{n}\right\|_{1,2} \rightarrow 0$ as $n \rightarrow \infty$ and such that

$$
w_{n}\left(\cdot+\tau_{n}\right)=\exp \circ \bar{\zeta}_{n}^{p} \quad \text { for all } n \text { large enough } .
$$

Using Theorem 4 and Lemma 5 we can give a proof of Theorem 3:

For $k \in \mathbb{N}$ define $\epsilon_{k}=\min (\epsilon(K), 1 / k)$ where $\epsilon(K)$ is as in Theorem 1. Let $\delta_{k}>0$ be a strictly decreasing sequence with $\delta_{k} \rightarrow 0$ as $k \rightarrow \infty$ and $\delta_{k} \leq \delta\left(u_{0}, v_{0}, \epsilon_{k}\right)$ for all $k \in \mathbb{N}$, where $\delta\left(u_{0}, v_{0}, \epsilon_{k}\right)$ is as in Theorem 4 (with $\epsilon$ replaced by $\epsilon_{k}$ ). By Lemma 5 , there is a strictly increasing sequence $n_{k} \in \mathbb{N}$, with $n_{k} \geq \bar{n}, k \in \mathbb{N}$, with $n_{k} \rightarrow \infty$ as $k \rightarrow \infty$ and such that

$$
\left\|\bar{\zeta}_{n}\right\|_{1,2} \leq \delta_{k}, \quad \text { for all } k \in \mathbb{N} \text { and } n \in \mathbb{N} \text { with } n_{k} \leq n<n_{k+1} .
$$

Let $n \geq n_{1}$ be arbitrary. Then there is a unique $k \in \mathbb{N}$ such that $n_{k} \leq n<n_{k+1}$. By Theorem 4 there is a $\left(u_{n}, v_{n}\right) \in M_{x, y} \times M_{y, z}$ such that

$$
\begin{aligned}
& u_{n}=\exp \circ \xi_{n}^{p} \text { for some } \xi_{n} \in H^{1,2}\left(u_{0}^{*} \mathcal{D}\right) \text { with }\left\|\xi_{n}\right\|_{1,2}<\epsilon_{k}, \\
& v_{n}=\exp \circ \eta_{n}^{p} \text { for some } \eta_{n} \in H^{1,2}\left(v_{0}^{*} \mathcal{D}\right) \text { with }\left\|\eta_{n}\right\|_{1,2}<\epsilon_{k}
\end{aligned}
$$

and

$$
w_{n}\left(\cdot+\tau_{n}\right)=\exp \circ \zeta_{n}^{p} \text { for some } \zeta_{n} \in H^{1,2}\left(\operatorname{preg}\left(u_{n}, v_{n}, \rho_{n}\right)^{*} \mathcal{D}\right) \text { with }\left\|\zeta_{n}\right\|_{1,2}<\epsilon_{k}
$$

and such that

$$
\zeta_{n} \in L_{\left(u_{n}, v_{n}, \rho_{n}\right)}^{\perp} .
$$

Since $\left\|\xi_{n}\right\|_{1,2} \rightarrow 0$ and $\left\|\eta_{n}\right\|_{1,2} \rightarrow 0$ as $n \rightarrow \infty$ and since $\left(u_{0}, v_{0}\right)$ lies in the interior of $K$ it follows, choosing $\bar{n}$ large enough, that $\left(u_{n}, v_{n}\right) \in K$ for all $n \geq \bar{n}$. Now the uniqueness part of Theorem 1 implies that

$$
\zeta_{n}=\xi_{\left(u_{n}, v_{n}, \rho_{n}\right)}
$$

where $\xi_{\left(u_{n}, v_{n}, \rho_{n}\right)}$ is as in Theorem 1 and so

$$
w_{n}\left(\cdot+\tau_{n}\right)=\operatorname{glue}\left(u_{n}, v_{n}, \rho_{n}\right) .
$$

Furthermore, using once again the fact that $\left\|\xi_{n}\right\|_{1,2} \rightarrow 0$ and $\left\|\eta_{n}\right\|_{1,2} \rightarrow 0$ as $n \rightarrow \infty$, we conclude that $u_{n}$ converges almost uniformly to $u_{0}$ and $v_{n}$ converges almost uniformly to $v_{0}$. This completes the proof. 
REMARKS. In Theorems 2 and 3 we actually obtain $C^{\infty}$-almost uniform convergence (defined in the obvious way). This follows from the fact that the functions considered there are solutions of a differential equation with a smooth right-hand side.

Note that if the interval $\left[\rho\left(u_{0}, v_{0}\right), \infty[\right.$ in the statement of Theorem 4 is replaced by an arbitrary compact interval $\left[\rho\left(u_{0}, v_{0}\right), \rho\left(u_{0}, v_{0}\right)+M\right]$, with $0<M<\infty$, then this theorem is a simple consequence of the usual tubular neighborhood theorem using the fact that $L^{\perp}$ is a vector bundle over $U(K) \times[\rho(K), \infty[$. The noncompactness of the interval $\left[\rho\left(u_{0}, v_{0}\right), \infty[\right.$ makes the proof more involved. Since the arguments are somewhat technical, we do not present them here, referring the interested reader to the paper [9] for details.

\section{References}

[1] S. Angenent and R. VAndervorst, preprint.

[2] V. Benci, A new approach to the Morse-Conley theory and some applications, Ann. Mat. Pura Appl. (4) 158, 1991, 231-305.

[3] C. C. Conley, Isolated Invariant Sets and the Morse Index, CBMS 38, AMS, Providence, 1978.

[4] K. Deimling, Nonlinear Functional Analysis, Springer Verlag, Berlin, Heidelberg, New York, 1985.

[5] S. K. Donaldson and P. B. Kronheimer, The Geometry of Four-Manifolds, Oxford University Press, 1990.

[6] A. Floer, Morse theory for Lagrangian intersections, J. Diff. Geometry 28, 1988, 513547.

[7] A. FLOER, An instanton-invariant for 3-manifolds, Commun. Math. Physics 118, 1988, $215-240$.

[8] A. Floer, Symplectic fixed points and holomorphic spheres, Commun. Math. Physics 120, 1989, 575-611.

[9] M. Rinaldi and K. P. RyBakowski, Tubular neighborhoods and the gluing principle in Floer homology theory, to appear.

[10] K. P. Rybakowski, On the homotopy index for infinite-dimensional semiflows, Trans. Amer. Math. Soc. 269, 1982, 351-382.

[11] K. P. Rybakowski, The Morse index, repeller-attractor pairs and the connection index for semiflows on noncompact spaces, J. Diff. Equations 47, 1983, 66-98.

[12] K. P. Rybakowski, The Homotopy Index and Partial Differential Equations, Springer Verlag, Berlin, Heidelberg, New York, 1987.

[13] K. P. Rybakowski and E. Zehnder, On a Morse equation in Conley's index theory for semiflows in metric spaces, Ergodic Theory Dyn. Systems 5, 1985, 123-143.

[14] M. Schwarz, Morse Homology, Birkhäuser Verlag, Basel, Boston, Berlin, 1993. 